\title{
Research on Knowledge Context Management Model Based on Ubiquitous Learning and Its Application
}

\author{
Ming Zeng \\ Hunan Modern Logistics College \\ Changsha, China
}

\begin{abstract}
In a ubiquitous learning environment, learners can use smart devices anytime, anywhere, and obtain the required knowledge points for learning in a massive knowledge base. However, the huge amount of knowledge resources in different scenarios and situations restricts the learners' learning and reduces the learning efficiency. This project focuses on the concentration of knowledge resources in different scenarios and the promotion of problems, and conducts basic scientific research on the knowledge management model that integrates knowledge context. Specifically, it includes the modeling of knowledge resource concentration and knowledge resource push, the construction and implementation of a Knowledge Context Management Model (KCMM) of integrated knowledge context, and the practical application of the model. It is hoped that the effective concentration of knowledge resources in the ubiquitous learning environment, the intelligent push of knowledge required based on different knowledge scenarios, and the accurate acquisition of knowledge content by learners will improve learning efficiency. It provides a theoretical basis for scene-based knowledge representation and knowledge management, and plays a dual inspiration at the theoretical and practical levels for guiding teaching resources and the construction of smart campuses.
\end{abstract}

Keywords-ubiquitous learning; knowledge management model; knowledge context; cyclic innovation

\section{INTRODUCTION}

Ubiquitous learning is often called U-learning. With the unprecedented development of information technology, Internet technology, and Internet of Things technology and its application in education, ubiquitous learning, as a new teaching method, has attracted widespread attention from educators. With this new learning method, learners can continuously and seamlessly obtain ubiquitous learning support without being limited by time, space and equipment. This effectively promotes more frequent flow of knowledge and more effective interaction between learners and knowledge. An important feature of ubiquitous learning is its autonomous learning. Learners use independent learning methods to learn knowledge according to different situations and needs. Knowledge exists in the environment like air resources, and becomes a kind of "on demand" resource. Therefore, ubiquitous learning requires massive, dynamically generated, cyclic and innovative learning resources to support it.

\section{RESEARCH BACKGROUND}

In recent years, space-based teaching has been in full swing, learning resources have been built and shared, and knowledge resources have skyrocketed. There are features of fragmentation, repetition and large amount. Due to different learning scenarios, to a certain extent, the differences in learners' search, discrimination, and summary capabilities reduce learning efficiency and restrict learning. Therefore, it is necessary to select high-quality knowledge resources from scattered and uneven network resources to meet the personalized learning of different learning individuals and learning organizations, to make content-rich and huge number of resource platforms more regular and orderly, and realize learners' scientific choice of knowledge, especially to better display knowledge resources under different devices, environments, and scenarios, and to realize the integration of context and knowledge. This will be the key to solving the problem of knowledge resource rule concentration and accurate intelligent push, and it is also a problem that education technology workers need to pay attention to and solve in the long run. Therefore, it is of great significance to study the knowledge context management model in the ubiquitous learning environment.

\section{AnAlysis of Research Status AND DEVELOPMENT TRENDS IN CHINA AND OTHER COUNTRIES}

\section{A. Current Situation of Ubiquitous Learning's Application}

The concept of universal learning abroad can be traced back to the concept of pervasive computing in 1988. After more than 20 years of development, it is currently in a relatively mature stage. Therefore, foreign studies on ubiquitous learning are in a leading position both in theory and in the application of technology. Vicky Jones et al. established a ubiquitous learning environment model based on the concepts of ubiquitous network technology and adaptive learning. The ubiquitous learning center, seamless interaction between students and devices, and communication between objects and devices are introduced. Some scholars have made a more in-depth analysis of the 
following items: 2004 U-Korea Master Policy Planning Project in Korea, Japan's "Oyako de Science", "Narikiri English" projects, and Harvard's "Wireless Handheld Device for Ubiquitous Learning" project and MIT's "Reliving the Revolution, RtR", etc. They also point out that the focus of foreign scholars' research lies in the close integration of ubiquitous computing and ubiquitous learning with practical application projects, focusing on highlighting actual scene changes and different situations.

In China, the study of ubiquitous learning started in 2003, and gradually began to develop. Up to the year of 2016, more than 100,000 articles related to "ubiquitous learning" have been retrieved from the Wanfang Data Academic Literature Database. Reading and analyzing the collected relevant literatures, it was found that the earliest was a coauthor of Bai Juan and Ge Shufang, and it was in a state of rapid growth in the following years. Feng Jingyu and others pointed out that since 2006. China has held special conferences on university study, which has promoted the enthusiasm for university study to a certain extent. For example, in October 2007, the third National Doctoral Forum on Educational Technology was held at Beijing Normal University to discuss topics such as "mobile learning and popular learning". In June 2008, the conference was hosted by the Shanghai Municipal Education Commission. In 2012, the Beijing Municipal Education Commission revealed that during the "Twelfth Five-Year Plan" period, the construction of a capital basic education cloud will be launched to provide students with pervasive "ubiquitous learning" services. All these have effectively promoted the development of U-Learning in China. With the deepening of research, ubiquitous learning can not only seamlessly connect the virtual world and the natural world, but also can turn to massive knowledge resource management and personalized adaptive situational awareness. Pan Jixin of North China University of Science and Technology and Yu Shengquan of Beijing Normal University have done a lot of research in these directions, providing important support for the development of domestic ubiquitous learning in China.

\section{B. The Current Status of Knowledge Management Under Ubiquitous Learning Environment}

The concept of knowledge management first appeared in business management in the $1980 \mathrm{~s}$. It is a management thought system based on the knowledge that "knowledge is valuable and can create value" in the era of knowledge economy. Early knowledge management revolved around the development of information technology. Therefore, the theoretical and practical activities of scholars and organizations mainly explore the application of information technology in knowledge management, and use information technology to manage the existing knowledge of enterprises. In 1985, Paul Strassman published "information profit", making people realize that knowledge can be identified and measured. In 1993, Dr. Vig published the first monograph on knowledge management, put forward the management of knowledge resources in the Internet environment, and made an important discussion on the needs of information environment learning. In 1997, Leif Edvinsson and Michael
S. Malone jointly published the authoritative work on Intellectual Capital, Exploring Hidden Intelligence, Realizing the Real Value of the Company, describing natural scenarios and all information that changes with the environment will become knowledge management object. Well-known economist and Nobel Prize winner Herbert Simon once pointed out that in the information age, the scarcest resource is not the information itself, but the ability to process information, especially the ability to process knowledge in pervasive computing. China's research on knowledge management started late, beginning in the 1990s, Chinese scholar Qiu Junping put forward the concepts of narrow knowledge management and broad knowledge management, and pointed out that narrow knowledge management refers to the management of knowledge itself. Broad knowledge management includes not only the management of knowledge itself, but also the management of various knowledge-related resources. Academician Wang Zhongtuo proposed the overall architecture of the knowledge system, including personnel, organization, technology, operation and culture. However, there is not much literature on knowledge management research based on ubiquitous learning environment. Taking Yan Yan as an example, he believes that the knowledge resources of ubiquitous learning are mainly based on Internet resources. In order to effectively store and manage huge amounts of knowledge information, ubiquitous learning emphasizes the relationship between learners and the environment with the support of ubiquitous computing environments, as well as strong interaction and situational awareness of learning. Pan Jixin and other scholars believe ubiquitous learning refers to a variety of learning activities where learners use accessible resources at any time and any place, based on their learning content and cognitive goals, with the support of context creation, ubiquitous technology, and ubiquitous computing. It is a process from quantitative change to qualitative change after digital learning and mobile learning have developed to a certain stage. Ubiquitous learning environment is a learning environment that integrates physical space and information space, natural space and intelligent space. It can provide an interoperable, universal and seamless learning system to realize the connection, integration and shared management of collaborators, learning content and learning services in the learning environment.

\section{Status of Knowledge Management Models Integrated Context}

The research of knowledge management model is carried out from the perspective of system and dynamics. The concept of knowledge management is three-dimensional. Time, knowledge characteristics and environment will be the most basic elements of this three-dimensional structure. The research of knowledge management mode is the process that people continue to deeply understand knowledge management. The 1991 SECI model proposed by Japanese scholar Nona is the most representative and basic model for foreign scholars. Research on the generation, transfer and reconstruction of organizational knowledge is considered by most scholars to be an effective "knowledge innovation" model. NoaKa analyzes the three levels of knowledge of 
individuals, teams and organizations from the perspective of explicit and tacit knowledge. Gunn divides knowledge into tacit and explicit types in the model research of knowledge type, form and level. But at the analysis level, he expanded the knowledge existing in individuals, organized the knowledge between teams and organizations, and made specific descriptions of the expression of knowledge at all levels. Carayannis proposed the concepts of metacognition, metalearning, and metaknowledge from the perspective of organizational learning, and further proposed the concept of organizational knowledge network, a knowledge management network that supports, monitors, acquires, evaluates, and continuously enriches the process of organizational cognitive ability. He also proposed a set of scientific evaluation systems, including the stages of knowledge generation, knowledge protection, knowledge transfer and knowledge regeneration, to promote the development and learning of "meta-knowledge". Based on the SECI model, Nonaka and Konno further proposed four kinds of "field", namely source development field, interaction field, network field, and practice field. The purpose of the "field" is to create an environment for knowledge exchange for the organization, to provide conditions for the generation of the "knowledge spiral", to study how to create a good organizational environment, to promote the process of knowledge innovation, and to actively create the corresponding "leaders" in the field. This is more effective than managing the knowledge transfer process directly. Wiig compares the comprehensive activities of knowledge analysis, processing, decision-making and management with the three pillars of the knowledge management framework, and elaborates the core content of knowledge management activities, including knowledge acquisition, knowledge value building, and active knowledge management. For China, although there are relatively many studies on knowledge creation and models that are closely related to knowledge management, most of them follow the SECI model proposed by foreign scholars Nonaka and Takeuchi, lacking their original thinking. In these studies, there are more theoretical discussions, less applied research, more surface research, and less deep research. Moreover, most scholars regard the SECI model as the truth and have not carefully considered their own inevitable value dilemma. Therefore, there is less research literature on the Knowledge Context Management Model. For example, Pan Xuwei and others proposed a KMIC model, and introduced the knowledge management index database integrated context and the knowledge management service integrated context into this model. It makes the knowledge management system context-sensitive, and finally analyzes the application significance of the model in knowledge management. Wang Jun focused on the in-depth analysis of key technologies in knowledge management of integrated knowledge situations. It can be seen that there are few studies on the knowledge management model of different scenarios and integrated situations in the ubiquitous environment, and it is necessary to make further academic discussions.

The scientific significance of this project lies in the indepth study and application of the Knowledge Context Management Model in the ubiquitous learning environment.
It will give detailed guidance on the practical level of resource construction and demonstration teaching in the famous teacher space of Hunan province, and help the construction of smart campus in colleges and universities has dual implications of theory and practice.

\section{KnOWledge ConteXt Management Model AND ITS APPLICATION}

\section{A. Research Ideas of Knowledge Context Management Model}

Through in-depth research on knowledge management and knowledge models for different scenarios and situations, this paper builds a general learning environment knowledge situation management model ("Fig.1") to solve the problem of concentration and push of knowledge resources in different scenarios and situations. It can help learners acquire knowledge accurately, quickly acquire knowledge, and improve learning efficiency. It lays the theoretical foundation for knowledge representation and knowledge management based on scenarios and situations, and provides method support for the technical implementation of some knowledge management systems. It can effectively guide the resource construction and management of famous teacher space, and provide theoretical and practical basis for further use in the construction of smart campus. 


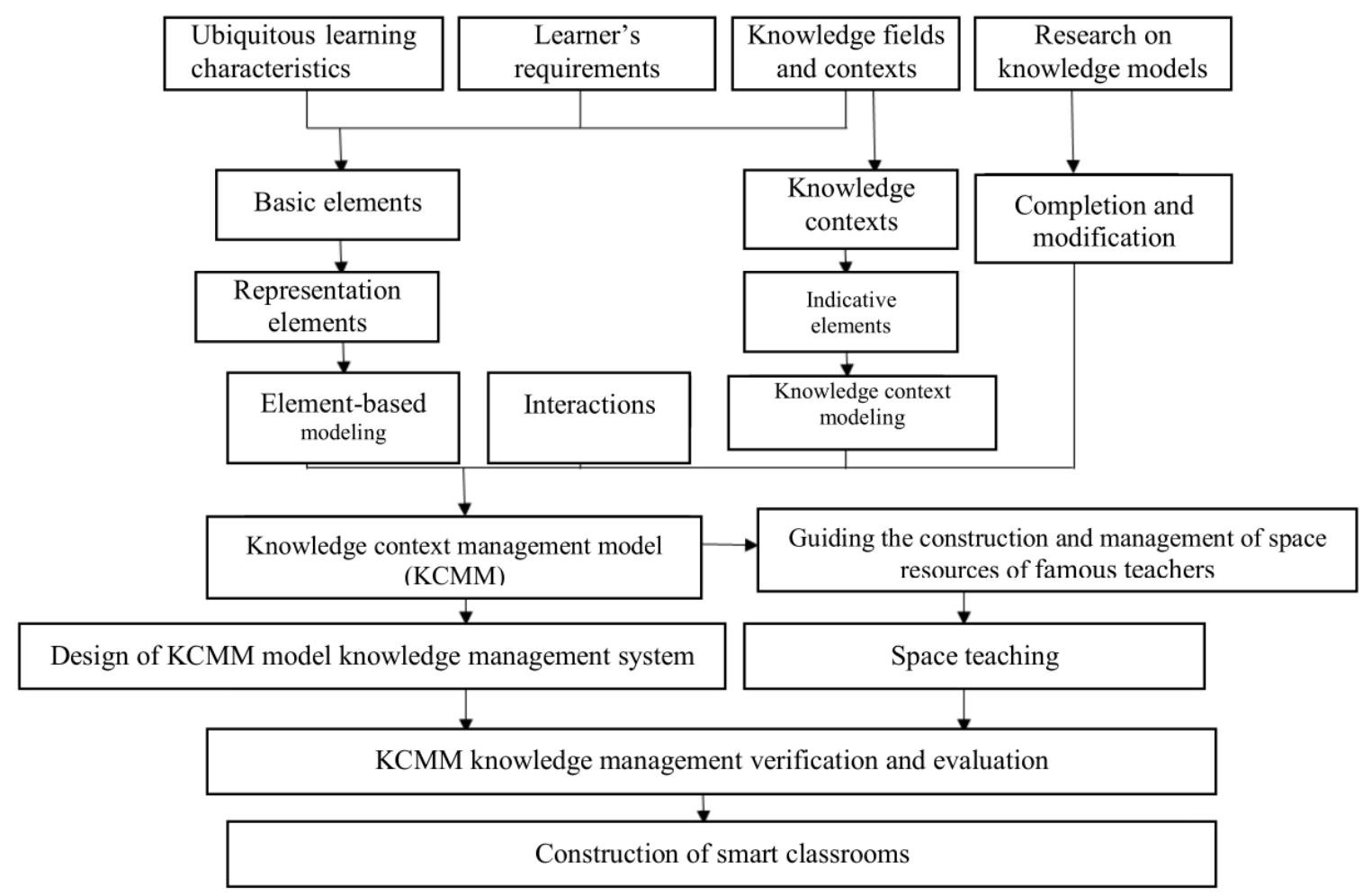

Fig. 1. Knowledge Context Management Model.

\section{B. Research Contents of Knowledge Context Management Model}

1) Research on integrated context knowledge management: It is based on the collection of ubiquitous learning characteristics to study the knowledge management needs in different contexts in the knowledge field, taking the key links of a series of cyclic innovation activities of knowledge as elements, further studying the relationship between the knowledge context and the integration of knowledge management and other factors.

2) Research on knowledge concentration and its modeling: It mainly studies the methods and rules of knowledge concentration in different learning scenarios in the ubiquitous learning environment, as well as the elements of knowledge concentration and the relationships between the factors.

3) Research on knowledge push and its modeling: It studies the selection of knowledge carriers, the methods of pushing, the rules, elements, and the relationships among elements, especially the representation elements of knowledge push, when integrating resources for knowledge push.

4) Research on knowledge context and its modeling: It mainly studies the process of occurrence of learners based on knowledge context when acquiring knowledge resources, as well as changes in the background, environment and scene of the process of knowledge acquisition, the relevant dimensions of the main features of the context model, the relationship between knowledge situation model and knowledge in the process of various knowledge activities, situation-based knowledge acquisition methods, and comparison and matching of situational similarities and representation elements of knowledge situations.

5) Implementation of knowledge context management model: According to the basic elements of integrated context knowledge management, the knowledge subject, knowledge concentration, knowledge push, knowledge context and its interaction process and interaction are combined to adopt a five-tuple model. That is: $\mathrm{KCMM}=$ ( $\mathrm{KA}, \mathrm{KF}, \mathrm{KP}, \mathrm{KC}, \mathrm{KR}$ ), $\mathrm{KA}$ is the subject of knowledge, $\mathrm{KF}$ is the knowledge concentration, $\mathrm{KP}$ is the knowledge push, $\mathrm{KC}$ is the knowledge context, and $\mathrm{KR}$ is the interaction between these elements. The four basic elements of knowledge management are integrated into one and combined with their functions, a knowledge context management model is constructed, and the unified modeling language UML specification is used to implement the model.

6) Application of knowledge context management model: It can use the KCMM model to construct and manage the resources of the famous teacher space, or design a knowledge management system for the KCMM model, verify and evaluate the model, or further use it to guide space teaching and smart campus construction.

\section{CONCLUSION}

In order to cope with the changing needs of ubiquitous learning equipment, scenarios, and environments, the existing knowledge management model needs to be further revised and improved. The theory of cyclic innovation of 
[8] Zhang Siming. Research on mobile learning resource design based on SECI model [D]. Master's thesis. 2014, Jinan: Shandong Normal University. (in Chinese)

knowledge can be incorporated into the knowledge management model to realize a knowledge management model that integrates knowledge context. It can be investigated to investigate the different learning needs of learners based on scenes and situations, the needs of learners for knowledge resources in the ubiquitous learning environment, and the context choices of new knowledge management models in applied research. Through a large amount of literature analysis, this paper summarizes the common features and shortcomings of existing knowledge management models, and proposes to build a new knowledge management model. The literature research method is used to analyze several common knowledge management reference models and to build a knowledge management model of cyclic innovation and integrated knowledge. By looking for the interaction between the knowledge elements, this paper proposes a knowledge management model of cyclical innovation and integrated knowledge context, integrating the personalized characteristics of the knowledge context in the ubiquitous learning environment to realize the construction of the knowledge context model. It can be used for individual learners' situational requirements and knowledge resource requirements. It is mainly used in the application research phase of the knowledge model. It collects data for actual application cases, and verifies and evaluates the KCMM model. It can build a knowledge management model of cyclic innovation and integrated knowledge context which helps learners acquire knowledge accurately, quickly master knowledge, improve learning efficiency, and provide a theoretical basis for knowledge representation and knowledge management based on scenarios and situations, strive for some progress in the application of this model, and provide theoretical and practical basis for further assisting the construction of smart campuses.

\section{REFERENCES}

[1] Wang Lei, Wu Chuangang. Multidimensional analysis of ubiquitous learning paradigm [J]. Journal of Mudanjiang Normal University (Philosophy and Social Science Edition), 2012, 168 (2): 131-132. (in Chinese)

[2] Y.Huang. P.Chiu. T.Liu. T.Chen.The design and implementation of a meaningful learning - based evaluation method for ubiquitous learning [J].Computers \& Education, 2011 (57) :2291-2302.

[3] Chen Min, Yu Shengquan, Yang Xianmin, Huang Kun. Design of content personalized recommendation model for ubiquitous learning [J]. Modern Educational Technology, 2011, 21 (6): 13-18. (in Chinese)

[4] Yang Xianmin, Yu Shengquan. Construction of Learning Resource Information Model in Ubiquitous Learning Environment [J]. China Educational Technology, 2010, 284 (9): 72-78. (in Chinese)

[5] Yang Xianmin. Research on the Orderly Evolution of Learning Resources in Ubiquitous Learning Environment [J]. China Educational Technology, 2015, 261 (1): 62-76. (in Chinese)

[6] Wang Jun. Construction of knowledge management model for ubiquitous learning environment and research on key technologies [J]. New Economy, 2014, 10 (2): 17-18. (in Chinese)

[7] Wu Xiaobo, Guo Wen, Liu Qinghua. Review of Research on Knowledge Management Model [J]. R\&D Management, 2002, 586 (4): 52-59. (in Chinese)
[9] Xu Huabin. Research on the Construction of Smart Campus in Colleges and Universities Based on Knowledge Cycle Model [D]. Master's Thesis. 2015, Dalian: Dalian University of Technology. (in Chinese)

[10] Jiang Qiang. Research on the support model of adaptive learning system and its implementation mechanism [D]. Doctoral Dissertation. 2012, Changchun: Northeast Normal University. (in Chinese)

[11] Yu Yan. Research on knowledge management in ubiquitous learning environment $[\mathrm{J}]$. Heilongjiang Science, 2016, 7 (7): 90-91. (in Chinese)

[12] Yu Yan. Research on knowledge management in ubiquitous learning environment [J]. Heilongjiang Science, 2016, 7 (7): 116-117. (in Chinese)

[13] Pan Jixin, Cheng Lulu. Theoretical Analysis and Construction of Knowledge Management Model in Ubiquitous Learning Environment [J]. Modern Educational Technology, 2011, 21 (10): 28-33 (in Chinese)

[14] Ma Haipeng. Research on a Learning Control Model in Ubiquitous Learning Environment [D]. Master's Thesis. 2014, Xi'an: Shaanxi Normal University. (in Chinese)

[15] Fu Daoming, Wu Wei. Construction of Knowledge Flow Model for Ubiquitous Learning Activities [J]. Distance Education Journal, 2016, 233 (2): 73-81. (in Chinese)

[16] Fan Qiaozhen. Research on Mobile Learning Model in the Ubiquitous Knowledge Environment [J]. Inner Mongolia Science Technology \& Economy, 2015, 323 (1): 158-159. (in Chinese)

[17] Pan Xuwei, Gu Xinjian, Cheng Yaodong, Li Jianming. Knowledge Context Management Model [J]. Computer Integrated Manufacturing System, 2006, 12 (2): 225-230. (in Chinese)

[18] Zhang Yi, Zhang Fan, Cheng Mingfeng, Du Chao. Construction of learning resource retrieval model based on context awareness in ubiquitous learning environment $[\mathrm{J}]$. China Educational Technology, 2010, 281 (6): 104-107. (in Chinese)

[19] Qiao M, Li J. Distance-based mixture modeling for classifi-cation via hypothetical local mapping. Statistical Analysisand Data Mining: The ASA Data Science Journal, 2016,9(1): 43-57

[20] Yang Xianmin, Yu Shengquan. Design of Ubiquitous Learning Environment from the Perspective of Ecology [J]. Educational Research, 2013, 3989 (3): 98-105 (in Chinese) 